\title{
O desafio da gestão de equipamentos médico-hospitalares no Sistema Único de Saúde
}

\author{
The challenge of managing medical equipment in the Unified Health \\ System
}

Aline Silva Amorim', Vitor Laerte Pinto Junior ${ }^{2}$, Helena Eri Shimizu ${ }^{\mathbf{3}}$

RESUMO O estudo analisou as políticas de gestão de equipamentos médico-hospitalares adotadas pelo Ministério da Saúde para o Sistema Único de Saúde. Realizou-se uma análise documental das publicações do Ministério da Saúde e uma análise de dados do Cadastro Nacional de Estabelecimentos de Saúde, no período de 2005 a 2013. O País instituiu uma Política de Gestão de Tecnologias em Saúde e uma área para a gestão dos equipamentos da Hemorrede. A oferta de equipamentos na rede privada é superior à pública, reforçando a necessidade da gestão e monitoramento de tecnologias para garantir o acesso aos usuários da rede pública e diminuir a dependência do Sistema Único de Saúde.

PALAVRAS-CHAVE Gestão em saúde; Sistema Único de Saúde; Tecnologia biomédica.

ABSTRACT The study analyzed the medical equipment management policies adopted by the Ministry of Health for the Unified Health System. We conducted a document analysis of the publications of the Ministry of Health and a data analysis of the National Registry of Health Facilities, in period from 2005 to 2013. The country established a Technology Management Policy on Health and an area for the management of Hemorrede equipment. The supply of equipment to the private network is superior to the public one, highlighting the need for management and monitoring technologies to ensure access to users in the public area and reduce the dependence of the Unified Health System.

KEYWORDS Health management; Unified Health System; Biomedical technology.

\footnotetext{
1 Universidade de Brasília (UnB) - Brasília, DF, Brasil. amorim.aline@ymail.com

2 Fundação Oswaldo Cruz (Fiocruz), Programa de Epidemiologia e Vigilância em Saúde (Pepivs) Brasília, DF, Brasil. vitorlaerte@fiocruz.br 


\section{Introdução}

O avanço da tecnologia e o crescimento da oferta de Equipamentos MédicoHospitalares (EMH) geraram um impacto financeiro nos Estabelecimentos Assistenciais de Saúde (EAS), ao investir em métodos mais sofisticados e caros.

O Sistema Único de Saúde (SUS) é composto por EAS de diferentes níveis de complexidade. Instituições privadas complementam os serviços do SUS e, inclusive, recebem investimentos, tendo preferência em relação às entidades filantrópicas e sem fins lucrativos, mediante contrato de direito público ou convênio (BRASIL, 1998). O SUS, portanto, também possui EMH instalados na rede privada financiados com recursos públicos.

O Ministério da Saúde (MS) utiliza a Portarian. ${ }^{0} 1.101 / \mathrm{GM}$ (Gabinete do Ministro), de 12 de junho de 2002, como base para o dimensionamento da assistência ambulatorial e alocação de EMH (BRASIL, 2002). Segundo Calil (2001), em 1985 não havia nenhum controle de equipamentos instalados no Brasil, tanto em termos locais como nacionais. Nesse contexto, a partir de 1990, o MS desenvolveu várias ações na área de $\mathrm{EMH}$, como a capacitação de recursos humanos Programa Pró-Equipo (BRASIL, 1992) -; a divulgação de ferramentas de gestão - Sistema para Planejamento e Dimensionamento de Equipamentos Médico-Hospitalares (BRASIL, 1991) - e aquisição de EMH para readequação da infraestrutura física e tecnológica do SUS - Projeto Reforço à Reorganização do SUS (ReforSUS). O ReforSUS ainda ofereceu uma capacitação a distância para os profissionais de saúde da rede pública: Gerenciamento da Manutenção de Equipamentos Médicos (Gema).

Entre 1999 e 2002, houve um aumento expressivo na capacidade instalada de EMH nos EAS públicos, variando de 31,3\% (tomógrafo computadorizado) a 135,8\% (aparelho de ultrassonografia) (IBGE, 2010). Tal aumento demonstrou a ampliação do investimento e a necessidade de se criar mecanismos mais eficientes de gestão.

Vários países executam ações visando à gestão eficiente dos parques tecnológicos instalados. As estratégias utilizadas incluem a elaboração de normas legais com vistas à regulamentação do financiamento e manutenção dos EMH, a criação de sistemas de informatizados de gestão, a formação de equipes técnicas regionais (fixas e móveis), entre outras. Esses fatores proporcionam a melhoria da qualidade dos serviços oferecidos à população, bem como a redução dos custos com novas aquisições e manutenções (GALVAN, 2004; GENTLES, 2004; GUTIÉRREZ, 2004; SUMALGY, 2004).

A gestão eficiente dos EMH é parte integrante dos cuidados ao paciente, sendo importante componente para garantia da integralidade, e está diretamente ligada à qualidade dos serviços. Assim, os objetivos deste estudo foram descrever a distribuição de equipamentos de diagnóstico por imagem, nas esferas federal, estadual, municipal e privada, e analisar os processos do MS envolvidos na gestão do parque tecnológico instalado no SUS.

\section{Metodologia}

Realizou-se uma análise documental para identificar quais são os mecanismos de gestão utilizados pelo MS na gestão do parque instalado no SUS, bem como uma análise quantitativa de dados disponíveis no Cadastro Nacional de Estabelecimentos de Saúde (CNES), de equipamentos de diagnóstico por imagem no âmbito público e privado, conforme apresentado a seguir. Foram pesquisados documentos oficiais do MS (Portarias, Resoluções, Manuais, Guias, Oficinas etc.) relacionados aos temas 'Gestão e Equipamento Médico-Hospitalar'. Os documentos selecionados tiveram os seguintes dados inseridos em uma matriz: ano de publicação, tipo de documento e o 
conteúdo abordado. No total, foram selecionados 11 documentos sobre os temas 'Gestão e Equipamentos Médicos', publicados no período de 2005 a 2013 pelo MS (quadro 1).

\begin{tabular}{|c|c|c|}
\hline Doc. no & Tipo e Data & Referências \\
\hline 1 & $\begin{array}{l}\text { Portaria - } \\
19 / 12 / 2005\end{array}$ & $\begin{array}{l}\text {. Ministério da Saúde. Portaria. Portaria MS no 2.510, de } 19 \text { de dezembro de } 2005 \text { - Instituiu comissão } \\
\text { interinstitucional para elaboração da Política de Gestão de Tecnologias em Saúde, sob coordenação da Secretaria de } \\
\text { Ciência, Tecnologia e Insumos Estratégicos (SCTIE). Diário Oficial [da] União. Brasília, DF, } 19 \text { dez. 2005. Disponível em: < } \\
\text { http://www.funasa.gov.br/site/wp-content/files_mf/Pm_2510_2005.pdf>. Acesso em: } 15 \text { abr. 2013. }\end{array}$ \\
\hline 2 & $\begin{array}{l}\text { Portaria - } \\
02 / 10 / 2007\end{array}$ & $\begin{array}{l}\text {. Ministério da Saúde. Portaria MS no 2.481, de } 2 \text { de outubro de } 2007 \text { - Institui o Sistema de Apoio à Elaboração } \\
\text { de Projetos de Investimentos em Saúde - SOMASUS. Diário Oficial [da] União. Brasília, DF, } 2 \text { out. 2007. Disponível em: } \\
\text { <http://bvsms.saude.gov.br/bvs/saudelegis/gm/2007/prt2481_02_10_2007.html>. Acesso em: } 15 \text { abr. } 2013 .\end{array}$ \\
\hline 3 & $\begin{array}{l}\text { Manual - } \\
2010\end{array}$ & $\begin{array}{l}\text { Ministério da Saúde. Secretaria de Ciência, Tecnologia e Insumos Estratégicos. Departamento de Ciência e } \\
\text { Tecnologia. Política Nacional de Gestão de Tecnologias em Saúde - PNGTS. Brasília, DF: Ministério da Saúde, 2010a. }\end{array}$ \\
\hline 4 & $\begin{array}{l}\text { Resolução } \\
\text { 25/01/2010 }\end{array}$ & $\begin{array}{l}\text {. Resolução da Diretoria Colegiada no 2, de } 25 \text { de janeiro de } 2010 \text { - Dispõe sobre o gerenciamento de tecnologias } \\
\text { em saúde em estabelecimentos de saúde. Diário Oficial [da] União. Brasília, DF, } 25 \text { jan. } 2010 \text { b. Disponível em: <http:// } \\
\text { bvsms.saude.gov.br/bvs/saudelegis/anvisa/2010/res0002_25_01_2010.html>. Acesso em: } 16 \text { abr. } 2013 .\end{array}$ \\
\hline 5 & $\begin{array}{l}\text { Manual - } \\
2011\end{array}$ & $\begin{array}{l}\text {. Ministério de Saúde. Secretaria Executiva. Departamento de Economia da Saúde e Desenvolvimento. Programação } \\
\text { Arquitetônica de Unidades Funcionais de Saúde. SOMASUS - Sistema de Apoio à Elaboração de Projetos de Investimentos } \\
\text { em Saúde. Brasília, DF: Ministério da Saúde, 2011c. (Atendimento Ambulatorial e Atendimento Imediato, v.1). Disponível } \\
\text { em: <http://bvsms.saude.gov.br/bvs/publicacoes/programacao_arquitetonica_somasus_v1.pdf> Acesso em: } 16 \text { abr. } 2015 \text {. }\end{array}$ \\
\hline 6 & $\begin{array}{l}\text { Portaria - } \\
12 / 12 / 2011\end{array}$ & $\begin{array}{l}\text {. Ministério da Saúde. Portaria MS no 2.915, de } 12 \text { de dezembro de } 2011 \text { - Institui a Rede Brasileira de Avaliação de } \\
\text { Tecnologias em Saúde (REBRATS). Diário Oficial [da] União. Brasília, DF, } 12 \text { dez. 2011a. Disponível em: <http://bvsms.saude. } \\
\text { gov.br/bvs/saudelegis/gm/2011/prt2915_12_12_2011.html>. Acesso em: } 15 \text { abr. } 2013 .\end{array}$ \\
\hline 7 & $\begin{array}{l}\text { Portaria - } \\
18 / 02 / 2011\end{array}$ & $\begin{array}{l}\text {. Ministério da Saúde. Portaria MS no 263, de } 18 \text { de fevereiro de } 2011 \text { - Institui Grupo de Assessoramento Técnico } \\
\text { em Gestão de Equipamentos dos Serviços de Hemoterapia e Hematologia Públicos, visando à elaboração de propostas } \\
\text { e pactuação de ações nas áreas de Gestão de Equipamentos nos Serviços De Hemoterapia e Hematologia Públicos. } \\
\text { Diário Oficial [da] União. Brasília, DF, } 18 \text { fev. } 2011 \text { b. Disponível em: <http://bvsms.saude.gov.br/bvs/saudelegis/gm/2011/ } \\
\text { prt0263_18_02_2011.html>. Acesso em: } 15 \text { abr. 2013. }\end{array}$ \\
\hline 8 & $\begin{array}{l}\text { Manual - } \\
2012\end{array}$ & $\begin{array}{l}\text {. Ministério da Saúde. Secretaria de Atenção à Saúde. Departamento de atenção especializada. Guia para elaboração } \\
\text { do Plano de gestão de equipamentos para serviços de Hematologia e Hemoterapia. Brasília, DF: Ministério da Saúde, } 2012 . \\
\text { Disponível em: <http://bvsms.saude.gov.br/bvs/publicacoes/elaboracao_plano_gestao_equipamentos_servicos_ } \\
\text { hematologia_hemoterapia>. Acesso em: } 10 \text { abr. } 2015 .\end{array}$ \\
\hline 9 & $\begin{array}{l}\text { Manual- } \\
2013\end{array}$ & $\begin{array}{l}\text { Ministério da Saúde. Secretaria de Ciência, Tecnologia e Insumos Estratégicos. Departamento de Ciência e Tecnologia. } \\
\text { Diretrizes metodológicas: elaboração de estudos para avaliação de equipamentos médicos assistenciais. Brasília, DF: Ministério da } \\
\text { Saúde, } 2013 a \text {. Disponível em: <http://bvsms.saude.gov.br/bvs/publicacoes/diretrizes_metodologicas_elaboracao_estudos. } \\
\text { pdf>. Acesso em: } 18 \text { abr. 2015. }\end{array}$ \\
\hline 10 & $\begin{array}{l}\text { Manual - } \\
2013\end{array}$ & $\begin{array}{l}\text { Ministério de Saúde. Secretaria Executiva. Departamento de Economia da Saúde e Desenvolvimento. Programação } \\
\text { Arquitetônica de Unidades Funcionais de Saúde. SOMASUS - Sistema de Apoio à Elaboração de Projetos de Investimentos } \\
\text { em Saúde. Brasília, DF: Ministério da Saúde, 2013b. (Programação Arquitetônica de Unidades Funcionais de Saúde, v. 2). }\end{array}$ \\
\hline 11 & $\begin{array}{l}\text { Manual - } \\
2013\end{array}$ & $\begin{array}{l}\text { Ministério de Saúde. Secretaria Executiva. Departamento de Economia da Saúde e Desenvolvimento. Programação } \\
\text { Arquitetônica de Unidades Funcionais de Saúde. SOMASUS - Sistema de Apoio à Elaboração de Projetos de Investimentos } \\
\text { em Saúde. Brasília, DF: Ministério da Saúde, 2013c. (Programação Arquitetônica de Unidades Funcionais de Saúde, v. 3). }\end{array}$ \\
\hline
\end{tabular}

Fonte: Elaboração própria

No CNES, os gestores de saúde de instituições públicas e privadas cadastram os equipamentos disponíveis na infraestrutura do SUS. A consulta ao CNES é disponibilizada no endereço eletrônico: http://www2.datasus. gov.br/DATASUS.

Este estudo se limitou a analisar o grupo de EMH de diagnóstico por imagens, 
equipamentos com alto custo de aquisição, infraestrutura e manutenção. Ressalta-se que a processadora radiológica não é um equipamento de diagnóstico, mas como foi inserido no grupo de Diagnóstico por Imagem (DIM) pelo MS, também foi considerado neste estudo. No campo Recursos Físicos do CNES, selecionou-se o campo Equipamentos, e na barra de opções 'Brasil, UF e Municípios'. O sistema apresentou três opções: Linha, Coluna e Conteúdo. Selecionou-se no campo Coluna a opção 'Equipamentos', no campo Linha 'Esfera Administrativa' e no campo Conteúdo ‘Disponíveis no SUS'. Foi realizada uma pesquisa com as opções anteriores para cada ano, considerando o período de dezembro de 2005 a dezembro de 2013.

\section{Resultados}

\section{Gestão do parque de EMH instalados no SUS}

No Brasil, a Portaria n. ${ }^{\circ} 2.510$, de 19 de dezembro de 2005, criou uma Comissão para elaborar a Política Nacional de Gestão de Tecnologias em Saúde (PNGTS) no âmbito do SUS, sendo que a sua coordenação foi exercida pelo Secretário de Ciência Tecnologia e Insumos Estratégicos, do MS (BRASIL, 2005). De acordo com essa portaria, tecnologias em saúde são: medicamentos, equipamentos, procedimentos técnicos, sistemas organizacionais, informacionais, educacionais e de suporte, programas e protocolos assistenciais por meio dos quais a atenção e os cuidados com a saúde são prestados à população. Em 2007, o MS formalizou, por meio da Portaria n. ${ }^{\circ}$ 2.481, o Sistema de Apoio à Elaboração de Projetos de Investimentos em Saúde (SomaSUS) (BRASIL, 2007). Essa ferramenta tem como objetivo auxiliar gestores e técnicos na elaboração de projetos de investimentos em infraestrutura na área de saúde, entre eles equipamentos médicos.

Após cinco anos da publicação da Portaria que institui a Comissão para Elaboração de Proposta de Política de Gestão de Tecnológica no âmbito do SUS (CPGT), o MS publicou o manual da PNGTS. Entre os propósitos dessa política, estão: a aquisição e monitoramento das tecnologias, o desenvolvimento de mapas regionais dos recursos tecnológicos existentes e o monitoramento das manutenções requeridas pela tecnologia para garantia de sua correta utilização e segurança dos usuários, dos profissionais e do meio ambiente. Houve, ainda, o incentivo para realizar cursos de pós-graduação e educação permanente, voltados para áreas relacionadas à gestão de EMH, tais como Avaliação de Tecnologias em Saúde (ATS) e Gerenciamento de Unidades de Saúde. Outro ponto observado foi a criação de mecanismos formais para articular os setores envolvidos no processo de gestão de tecnologias em saúde. $\mathrm{O}$ monitoramento de tecnologias em saúde foi definido nessa portaria como um

\footnotetext{
processo sistemático e contínuo de acompanhamento do uso da tecnologia, visando à obtenção de informações em tempo oportuno para subsidiar a tomada de decisão, relativas à substituição, ao abandono ou à ampliação de cobertura. (BRASIL, 2010B).
}

A Agência Nacional de Vigilância Sanitária (Anvisa) instituiu a Resolução da Diretoria Colegiada (RDC) n. ${ }^{\circ}$ 2, de 25 de janeiro de 2010, que "Dispõe sobre o gerenciamento de tecnologias em saúde em estabelecimentos de saúde”. Ela teve como objetivo

\footnotetext{
estabelecer os critérios mínimos a serem seguidos pelos estabelecimentos de saúde para o gerenciamento de tecnologias em saúde utilizadas na prestação de serviços de saúde, de modo a garantir a sua rastreabilidade, qualidade, eficácia, efetividade e segurança. (BRASIL, 2010B).
}

Essa resolução se aplica aos "estabelecimentos de saúde em âmbito hospitalar, ambulatorial e domiciliar e àqueles que prestam serviços de apoio ao diagnóstico e terapia, intra ou extra-hospitalar" (BRASIL, 2010B), reconhecendo como equipamentos de saúde: 
[...] o conjunto de aparelhos e máquinas, suas partes e acessórios utilizados por um estabelecimento de saúde onde são desenvolvidas ações de diagnose, terapia e monitoramento, bem como os equipamentos de apoio, os de infraestrutura, os gerais e os médico-assistenciais. (BRASIL, 2010B).

Considera-se o gerenciamento

[...] desde o planejamento e entrada no estabelecimento de saúde até seu descarte, visando à proteção dos trabalhadores, a preservação da saúde pública e do meio ambiente e a segurança do paciente. (BRASIL, 2010B).

Dessa forma, os gestores são orientados a elaborar um Plano de Gerenciamento (PG) para as tecnologias abrangidas por esse regulamento técnico. Caso esse serviço seja terceirizado, "não isenta o estabelecimento de saúde contratante da responsabilização perante a autoridade sanitária”. Com relação à infraestrutura física para a realização das atividades de gerenciamento de tecnologias em saúde, a resolução orienta que esta deve ser compatível com a RDC n. ${ }^{\circ} 50$, de 21 de fevereiro de 2002, da Anvisa (BRASIL, 2010B).

Em 2011, o MS publicou o primeiro manual do SomaSUS, dando continuidade ao trabalho iniciado em 2007 com a publicação da Portaria n. ${ }^{\circ}$ 2.481. No primeiro volume da série, o tema desenvolvido foi 'Unidades de Atendimento Ambulatorial e Atendimento Imediato'. Trata-se de um manual ilustrado contendo os ambientes dos EAS, com seus respectivos equipamentos e mobiliários - exceto material de consumo. O objetivo dessa publicação é "fornecer informações técnicas estratégicas para a elaboração de projetos de investimentos, em particular relativos à execução de obras e à aquisição de equipamentos médico-hospitalares" (BRASIL, 2013B, P. 7). Nesse mesmo ano, foi criada a Rede Brasileira de Avaliação de Tecnologias em Saúde (Rebrats) com a finalidade de buscar por qualidade e excelência na conexão entre pesquisa, política e gestão, por meio da elaboração de estudos de avaliação de tecnologias em saúde, nas fases de incorporação, monitoramento e exclusão de tecnologias no âmbito do SUS. (BRASIL, 2011A).

Ao analisar os documentos, verificou-se que a Coordenação-Geral de Sangue e Hemoderivados (CGSH/MS) instituiu em 2011 o Grupo de Assessoramento Técnico em Gestão de Equipamentos dos Serviços de Hemoterapia e Hematologia (GAT). Foram atribuídas as seguintes atividades ao GAT: elaboração de propostas na área de gestão de equipamentos nos Serviços de Hemoterapia e Hematologia, assessoria para a CGSH/MS no que se refere à gestão de equipamentos e estabelecimento de um modelo de gestão de equipamentos. Essas atividades estavam restritas aos EAS que compõem a Hemorrede (Rede de Hemoderivados) (BRASIL, 2011C).

A análise do Doc. 7 revelou as diversas ações desenvolvidas pelo GAT, como oficinas de capacitação na área de engenharia clínica e capacitação de profissionais da Hemorrede para a utilização do software Sistema de Gerenciamento de Equipamentos para a Hemorrede Pública Nacional (Hemosige). Além disso, foi criada uma subárea de Gestão de Equipamentos no organograma da Gestão Financeira e Assessoria Técnica (GFAT), da CGSH, cuja missão é "assessorar tecnicamente a Rede de Hemocentros Públicos do País e a própria CGSH no desenvolvimento das ações inerentes à gestão de equipamentos” (BRASIL, 2011B). A GFAT ficou responsável por:

[...] nortear os investimentos realizados pelo MS em inovação tecnológica; aumentar a vida útil do parque de equipamentos; conhecer o estado de funcionamento dos equipamentos localmente; gerenciar os contratos de manutenção dos equipamentos; avaliar a aquisição de novos equipamentos; orientar a qualificação de equipamentos; e validar os processos. (BRASIL, 2011B). 
Dois volumes do SomaSUS foram publicados em 2013: o Volume 2 - Internação e Apoio ao Diagnóstico e à Terapia (Reabilitação); e o Volume 3 - Apoio ao Diagnóstico e à Terapia (Imagenologia) (BRASIL, 2013A, 2013B), ambos também baseados na RCD n. ${ }^{\circ}$ 50/2002.

Por fim, em 2013, o MS publicou o manual 'Diretrizes metodológicas: elaboração de estudos para avaliação de equipamentos médicos assistenciais', cujo objetivo é

nortear a análise de técnicos e gestores interessados na avaliação de equipamentos médicos assistenciais (EMA), seja na inclusão, modificação ou exclusão dessas tecnologias nos diversos níveis de gestão do Sistema único de Saúde (SUS). (BRASIL, 2013A, P. 13).

O manual apresentou as práticas de ATS para EMA, considerando seis domínios: Clínico, Admissibilidade, Técnico,
Operacional, Econômico e Inovação. A avaliação é uma etapa importante na gestão de $\mathrm{EMH}$, pois garante uma aquisição eficiente com ganhos para o EAS e o usuário.

\section{Equipamentos: rede pública $x$ rede privada}

O CNES desenvolvido em 2000 é a ferramenta do MS para disponibilizar informações das atuais condições de infraestrutura de funcionamento dos EAS do SUS, inclusive de equipamentos. A coleta dos dados acerca dos EMH do grupo de (DIM) instalados na rede pública nas três esferas (federal, estadual e municipal) apresentou o total de 15.217 equipamentos e 37.610 na rede privada. A tabela 1 apresenta a quantidade de equipamentos cadastrados no CNES, na rede pública e privada, no período de 2005 a 2006.

Tabela 1. Relação de equipamentos de diagnóstico por imagem e terapia da rede pública (municipal, estadual e federal) e privada, cadastrados no CNES no período de 2005 a 2013

\begin{tabular}{|c|c|c|c|c|c|c|c|c|c|}
\hline Tipo de equipamento & 2005 & 2006 & 2007 & 2008 & 2009 & 2010 & 2011 & 2012 & 2013 \\
\hline Aparelho de Densitometria Óssea - PR* & 924 & 1000 & 1068 & 1146 & 1263 & 1356 & 1438 & 1498 & 1590 \\
\hline Aparelho de Densitometria Óssea - PU** & 35 & 40 & 41 & 61 & 67 & 77 & 80 & 85 & 99 \\
\hline Aparelho de Hemodinâmica - PR & 245 & 266 & 284 & 313 & 343 & 365 & 375 & 396 & 398 \\
\hline Aparelho de Hemodinâmica público - PU & 74 & 79 & 83 & 87 & 90 & 90 & 92 & 93 & 104 \\
\hline Aparelho de Raios X com Fluoroscopia - PR & 651 & 658 & 671 & 689 & 706 & 708 & 681 & 683 & 688 \\
\hline Aparelho de Raios X com Fluoroscopia - PU & 240 & 254 & 256 & 268 & 277 & 274 & 276 & 284 & 292 \\
\hline Aparelho de Raios X de 100 a 500 mA - PR & 8062 & 8652 & 8888 & 9388 & 9862 & 10217 & 13041 & 10503 & 10762 \\
\hline Aparelho de Raios X de 100 a 500 mA - PU & 4767 & 4962 & 5061 & 5384 & 5595 & 5873 & 6078 & 6356 & 6525 \\
\hline Gama Câmara PR & 423 & 441 & 469 & 494 & 519 & 548 & 559 & 585 & 603 \\
\hline Gama Câmara PU & 89 & 88 & 96 & 109 & 121 & 116 & 121 & 132 & 127 \\
\hline Mamógrafo com comando simples - PR & 1667 & 1813 & 1934 & 2069 & 2242 & 2421 & 2479 & 2484 & 2594 \\
\hline Mamógrafo com comando simples - PU & 99 & 100 & 108 & 127 & 137 & 144 & 142 & 145 & 150 \\
\hline Mamógrafo com estereotaxia - PR & 401 & 435 & 459 & 495 & 538 & 576 & 589 & 580 & 596 \\
\hline Mamógrafo com estereotaxia - PU & 89 & 93 & 90 & 104 & 111 & 125 & 129 & 127 & 121 \\
\hline Mamógrafo Digital - PR & 0 & 0 & 0 & 0 & 0 & 0 & 0 & 19 & 37 \\
\hline Mamógrafo Digital - PU & 0 & 0 & 0 & 0 & 0 & 0 & 0 & 30 & 50 \\
\hline Processadora para Mamógrafo - PR & 0 & 0 & 0 & 0 & 0 & 0 & 133 & 518 & 594 \\
\hline Processadora para Mamógrafo - PU & 0 & 0 & 0 & 0 & 0 & 0 & 38 & 180 & 205 \\
\hline Ressonância Magnética - PR & 377 & 440 & 506 & 616 & 748 & 851 & 971 & 1107 & 1258 \\
\hline
\end{tabular}




\begin{tabular}{|c|c|c|c|c|c|c|c|c|c|}
\hline \multicolumn{10}{|l|}{ Tabela 1. (cont.) } \\
\hline Ressonância Magnética - PU & 29 & 35 & 43 & 50 & 58 & 65 & 78 & 80 & 99 \\
\hline Tomógrafo Computadorizado - PR & 1197 & 1281 & 1365 & 1524 & 1690 & 1860 & 1940 & 2116 & 2311 \\
\hline Tomógrafo Computadorizado - PU & 233 & 253 & 277 & 309 & 341 & 373 & 394 & 425 & 463 \\
\hline Ultrassom Diagnóstico - PR & 12558 & 13347 & 13757 & 14454 & 15170 & 15772 & 15979 & 16164 & 16568 \\
\hline Ultrassom Diagnóstico - PU & 3419 & 3747 & 4077 & 4511 & 4998 & 5442 & 5874 & 6234 & 6593 \\
\hline Total por ano & 35579 & 37984 & 39533 & 42198 & 44876 & 47253 & 51487 & 50824 & 52827 \\
\hline
\end{tabular}

Fonte: Elaboração própria

$\mathrm{PR}^{\star}=$ Rede privada; $\mathrm{PU}^{\star \star}=$ Rede pública

O gráfico 1 apresenta a quantidade total de equipamentos de diagnóstico por imagem instalados na rede pública e rede privada. Ressalta-se que o ano de cadastramento no CNES pode não corresponder ao ano de aquisição e instalação dos equipamentos, uma vez que essa ação é realizada pelos gestores.

Gráfico 1. Equipamentos do grupo de diagnóstico por imagem da rede pública e privada, cadastrados no CNES no período de 2005 a 2013

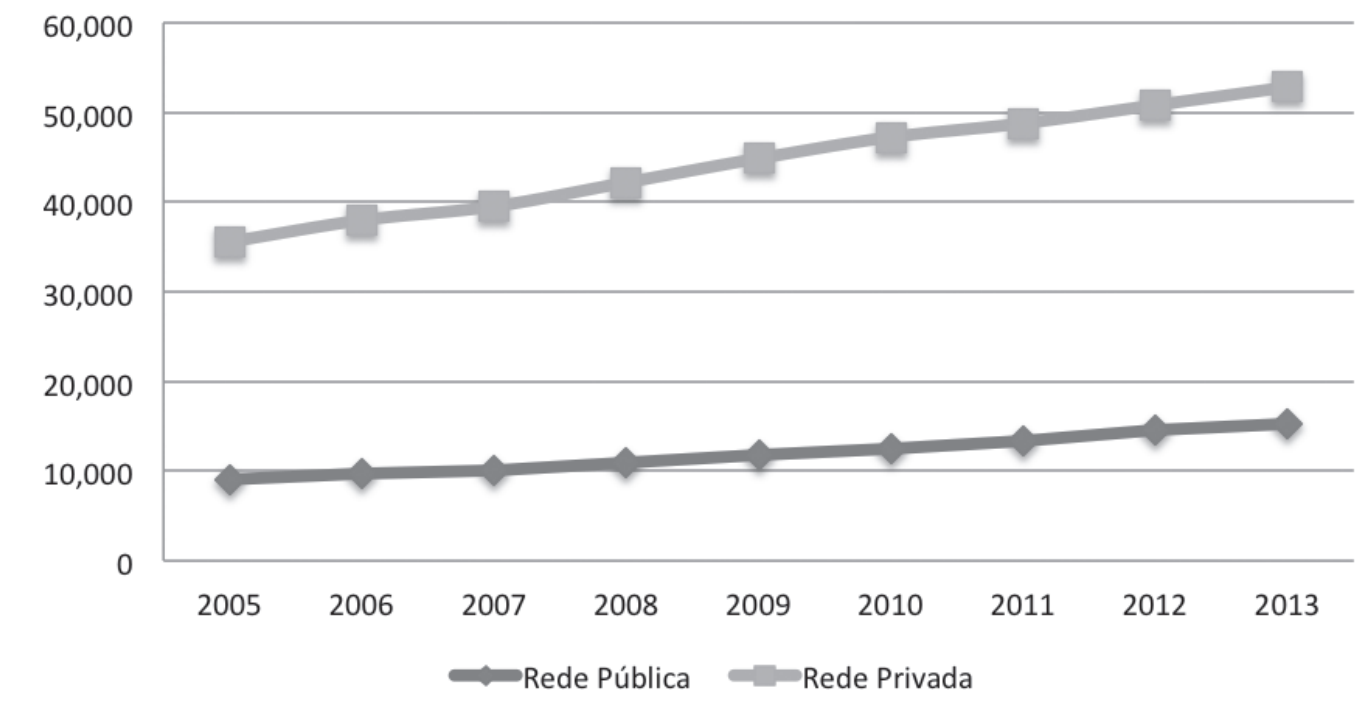

Fonte: Elaboração própria

\section{Discussão}

Apesar de vários programas para a aquisição de EMH terem sido desenvolvidos ao longo dos anos no Brasil, somente em 2005 foi criada uma Comissão para elaborar a PNGTS. Segundo Guimarães (2008), no
País, a maior preocupação está concentrada na compra de equipamentos, e, por uma questão cultural, a operação e manutenção são negligenciadas. Isso resulta na baixa qualidade da prestação de serviços, atrasos na instalação e interrupções no funcionamento dos equipamentos. 
A aquisição de EMH é responsabilidade dos estados e municípios, porém, o MS desenvolveu ferramentas para auxiliar os gestores nessa atividade. A ferramenta SomaSUS assemelha-se ao Sisplan, implantado em 1994 pelo MS, porém, mais detalhado. Nos três volumes publicados, não foram identificadas orientações sobre a gestão dos equipamentos ou mesmo espaço físico destinado para esta finalidade - conforme Doc. 4 . Embora não seja o foco dos manuais, propõe-se a abordagem desses assuntos para que os gestores sejam estimulados a desenvolver essas atividades nos EAS. Orientações para o cadastramento dos EMH (pós-aquisição) no CNES não foram abordadas em nenhum dos volumes analisados. Dessa forma, sugere-se que tal orientação também seja inserida nos manuais.

Dependendo do porte do equipamento, há necessidade de construir ou readequar a infraestrutura física, os recursos humanos especializados, tratamento de resíduos diferenciado, manutenções periódicas, insumos e substituição de peças de alto custo. Nesse aspecto, a Colômbia implementou em 1997 a Lei da Manutenção (LM). De acordo com esta Lei, as instituições públicas devem reservar $5 \%$ do seu orçamento para a manutenção da infraestrutura física e tecnológica. Essas instituições ainda devem apresentar de maneira compulsória um Plano de Manutenção (PM) anual, evidenciando os custos com a aquisição e manutenção de seus equipamentos (GUTIÉRREZ, 2004).

O manual da PNGTS relatou que cabe ao MS apoiar os gestores na "implantação das tecnologias e no seu monitoramento e manutenção após a incorporação" (BRASIL, 2010A, P. 23). Não ficou claro, contudo, se o MS irá monitorar as falhas técnicas ou quebras dessas tecnologias (equipamentos) instaladas nos EAS e como essas ações seriam realizadas. Além disso, o MS reconhece a necessidade de capacitação dos gestores e da estruturação do próprio MS nessa área quando relatou a necessidade de "formação de um quadro permanente de pessoal qualificado para atender à necessidade na área de gestão de tecnologias em saúde" (BRASIL, 2010A, P. 22) De maneira geral, observa-se que a PNGTS considerou o tipo de tecnologia a ser inserida no SUS, por exemplo, a substituição de uma tecnologia analógica por uma digital. Portanto, não foram identificadas ações voltadas para a gestão de EMH instalados no âmbito do SUS, embora eles estejam incorporados no conceito de tecnologia em saúde. A ferramenta CNES também não foi citada nessa política.

No Canadá, existem serviços regionais de engenharia clínica, em que uma equipe com cerca de 63 profissionais coordena até seis hospitais (GENTLES, 2004). Essas equipes atuam na gestão do parque instalado e desenvolvem atividades envolvendo a escolha das tecnologias adequadas, logística, instalação, capacitação de usuários, manutenção e baixa de patrimônio, entre outras. O Paraguai reformulou o seu sistema de saúde com base em dois componentes: ATS e Gerenciamento de Tecnologia (GT). Hospitais e centros de referência com o mínimo de 100 leitos devem ter uma equipe de manutenção, oficinas de manutenção são distribuídas de acordo com a demanda dos hospitais e oficinas móveis dão suporte aos hospitais localizados em áreas remotas ou rurais (GALVAN, 2004). Considerando-se a extensão territorial do Brasil, essa alternativa pode contribuir para a melhoria da qualidade de unidades de saúde de municípios do interior, principalmente nas regiões mais carentes.

Acredita-se que a publicação do Doc. 4, pela Anvisa, se deu pelo fato desse órgão ser responsável pela autorização de funcionamento dos EAS, controle e fiscalização sanitária de equipamentos. Essa resolução orienta o gestor na definição e padronização de critérios para as etapas de gerenciamento de tecnologias em saúde, inclusive, na elaboração de um Plano de Manutenção. Em comparação com a Colômbia, que implementou a LM e o PM compulsórios para suas unidades 
de saúde em 1997, o Brasil está defasado em 13 anos (GUtIÉRREZ, 2004). Estudos detalhados são necessários para analisar a viabilidade das instituições brasileiras em reservar parte do seu orçamento para a manutenção da infraestrutura física e tecnológica, uma vez que são conhecidas na literatura as deficiências no financiamento da saúde nacional. Por outro lado, o descumprimento das disposições contidas na resolução da Anvisa constitui uma infração sanitária. Verificou-se que não há relatos sobre a percepção por parte da Anvisa ou do MS para que esses estabelecimentos repassem dados do seu parque de equipamentos para um sistema de 'gestão' nacional ou mesmo para o CNES. Tal fato demonstra a desarticulação entre os órgãos e áreas internas do MS em relação às ações referentes aos EMH instalados no SUS.

Exceto o CNES, não existem sistemas informatizados que avaliem a situação do parque de equipamentos instalados no SUS. Moçambique era afetado pela falta da cultura de manutenção, de recursos escassos (físicos e materiais), falta de especialistas, processos de doação e uso de tecnologias inapropriadas. Ante esse cenário, um Sistema de Informação (SI) foi implantado para a obtenção de indicadores relacionados ao inventário e manutenção (exceto a preventiva) dos equipamentos instalados naquele país (SUMALGY, 2004).

Ao contrário do sistema canadense, em que a terceirização da gestão de EMH não é comum (GENTLES, 2004), no Doc. 4 foram citadas as condições para a adoção dessa medida. De acordo com o Art.7, a "terceirização do serviço pode ser realizada desde que não haja nenhum impedimento legal, devendo a terceirização obrigatoriamente ser feita mediante contrato formal" (BRASIL, 2010B), mas, isso "não isenta o EAS contratante da responsabilização perante a autoridade sanitária” (BRASIL, 2010B). Nesse caso, o controle deve ser mais rígido devido a conflitos de interesse existentes nessa área. A manutenção de equipamentos de DIM geralmente é realizada pelo fabricante e envolve contrato de manutenção periódica do equipamento. Esse é outro ponto que pode ser explorado em trabalhos futuros: quanto se gasta com contratos desse tipo no SUS?

A Rebrats foi um ganho para o SUS, mas os trabalhos envolvendo a avaliação de EMH ainda são escassos. Suas atividades são direcionadas para a avaliação e o monitoramento de novas tecnologias a serem introduzidas no SUS, principalmente medicamentos.

Identificou-se nos documentos analisados que somente a CGSH realiza gestão de equipamentos, no âmbito do MS. Ao criar uma área para GE, a CSGH desenvolveu várias atividades junto aos gestores dos hemocentros (oficinas de treinamento, desenvolvimento de software de gestão de equipamentos, entre outros). Tal medida, além de permitir um controle detalhado da situação atual do parque instalado na Hemorrede, garante o planejamento e a aplicação de políticas efetivas na área de hemoderivados. De acordo com Doc. 8, os gestores da Hemorrede foram capacitados para gerir tecnologias e operar o sistema informatizado Hemosige. Acreditase que o tamanho do parque tecnológico da Hemorrede tenha favorecido o desenvolvimento dessas atividades.

A publicação Doc. 9 relatou que está atendendo a PNGTS e tem como finalidade incentivar o desenvolvimento de atividades de gestão de EMH no âmbito dos SUS. Nessa publicação, o SomaSUS e a RDC n. ${ }^{\circ}$ 2/2010 são considerados. $\mathrm{O}$ documento ainda evidencia as considerações a serem feitas na aquisição de EMH (infraestrutura, manutenção, recursos humanos etc.). Isso demonstra que o MS vem buscando desenvolver ferramentas para auxiliar os gestores na área de EMH.

O quantitativo de EMH de DIM neste estudo teve como objetivo conhecer o tamanho do desafio a ser enfrentado pelos gestores no MS, pois, conforme relatado na PNGTS, é responsabilidade desse ministério desenvolver ações para o monitoramento das 
tecnologias - entre elas, EMH - do SUS. Os dados demonstraram que o País possui cerca de 15 mil equipamentos de DIM instalados no SUS, sendo que estão sob responsabilidade da instituição receptora (municipal, estadual ou federal).

O SUS herdou uma estrutura física e tecnológica sucateada do antigo Instituto Nacional de Assistência Médica da Previdência Social (Inamps). Segundo a Lei n. ${ }^{\circ} 8.689$, de 27 de julho de 1993, que extinguiu o Inamps, os bens imóveis e o acervo físico, documental e material integrantes do seu patrimônio deveriam ser inventariados e incorporados ao patrimônio da União dentro de 180 dias (BRASIL, 1993). Esses bens ficaram sob a responsabilidade do MS e poderiam ser doados ou cedidos a municípios, estados e Distrito Federal para utilização em hospitais e postos de assistência à saúde, desde que especificados os destinatários e sua utilização. Nesse contexto, o MS implantou vários programas para readequar a infraestrutura tecnológica do SUS, principalmente na área de média e alta complexidade, como o ReforSUS (BRASIL, 1993).

Toscas (2013) relatou que os equipamentos de maiores valores modais financiados pelo MS são do grupo DIM, tais como: Ressonância Magnética (RM), Tomógrafo Computadorizado (TC), Arco Cirúrgico (AC) e os Mamógrafos (Analógicos e Digitais) (MA). Esse mesmo estudo apontou um aumento significativo do investimento do MS em EMH ao longo dos anos: $150 \%$ no período de 2010 a 2011 e $80 \%$ no período de 2011 a 2012.

O mamógrafo digital é o equipamento com maior quantitativo em relação à área privada. Uma hipótese para tal fato foi a implantação da Política Nacional de Redução do Câncer de Mama (Decreto n. ${ }^{\circ} 7.508$, de 28 de junho 2011). Embora os equipamentos de RM, TC e MA sejam aqueles mais solicitados pelas instituições (TOSCAS, 2013), a quantidade instalada na rede pública ainda é muito inferior à privada (gráfico 1).
Gutierrez (2009) analisou a oferta de TC para o tratamento do Acidente Vascular Cerebral Agudo (AVC) no Brasil. Os resultados apontaram uma forte presença da esfera privada com $87,4 \%$ dos tomógrafos existentes no País e 73,4\% dos tomógrafos disponíveis ao SUS, além da alocação de equipamentos pouco equitativa e a concentração de TC nos municípios mais populosos e de melhor situação socioeconômica.

Em 2005, o Instituto Brasileiro de Geografia e Estatística (IBGE) realizou um estudo sobre o parque de equipamentos brasileiro. De acordo com a pesquisa, o País tinha 39.254 equipamentos, um aumento de $20 \%$ com relação ao ano anterior (ANDREAZZI, 2009). Os equipamentos de Raios $X$ apresentavam $45 \%$ do total de equipamentos e aqueles que apresentaram maior variação no período analisado foram: ressonância magnética (93\%); mamógrafo com comando simples (71\%); ultrassom doppler colorido (58\%); e Raios X para Hemodinâmica (51\%). Ainda se observou uma maior variação do quantitativo da rede pública em relação à privada, resultado do Projeto ReforSUS, que teve um papel importante na alocação de EMH no SUS (BRASIL, 2010). Embora o MS tenha investido na rede pública, a rede privada apresentou um maior quantitativo nesse mesmo período (gráfico 1). Além disso, a rede privada investe em infraestrutura e recursos humanos para gerir o seu patrimônio, uma vez que um equipamento sem funcionamento acarreta prejuízos financeiros para a instituição.

Outro ponto relevante que deve ser revisto pelo MS é a limitação do CNES. Esse banco de dados apresenta somente alguns equipamentos financiados pelo Fundo Nacional de Saúde (FNS), de um total de aproximadamente 840 itens. O único grupo de equipamentos completo é o DIM, com 17 tipos de equipamentos cadastrados, talvez devido ao alto custo do investimento (equipamento, infraestrutura física e recursos humanos). Recentemente, o equipamento tomógrafo computadorizado com tecnologia de 
emissão de pósitrons (PET-CT) foi inserido no CNES, constando 18 equipamentos cadastrados, estando 13 equipamentos disponíveis ao SUS. Sabe-se que esse número é superior ao informado no CNES, mas, conforme relatado, essa informação depende do gestor.

Segundo com Gomez (2004), no Equador, as informações sobre inventários econômico e técnico dos equipamentos instalados eram insuficientes e os hospitais não tinham acesso à internet. O País redefiniu as ações e implantou um modelo semelhante ao utilizado pelo Canadá. Na Estônia, o inventário de equipamentos foi reorganizado, padronizado e centralizado no sistema nacional de saúde, permitindo uma série de mudanças regulatórias e legislativas (AID; GOLUBJATNIKOV, 2004).

\section{Conclusão}

Conclui-se, com base na documentação analisada, que o MS não dispõe de um sistema informatizado ou ferramenta para gerir os

\section{Referências}

AID, S., GOLUBJATNIKOV, O. Clinical Engineering in Estonia. In: DYRO, J. (Org.). The Clinical Engineering Handbook. New York: Elsevier, 2004, p. 80-81.

ANDREAZZI, M. A. R.; ANDREAZZI, M. F. S. Escassez e fartura: distribuição da oferta de equipamentos de diagnóstico por imagem no Brasil. In: INSTITUTO BRASILEIRO DE GEOGRAFIA E ESTATÍSTICA (IBGE). Indicadores sociodemográficos e de saúde no Brasil. Rio de Janeiro: IBGE, 2009. Disponível em: < http:// www.ibge.gov.br/home/estatistica/populacao/indic_sociosaude/2009/com_esca.pdf>. Acesso em: 20 abr. 2014.

APARELHOS DE ÚLTIMA GERAÇÃO estão encaixotados no hospital da UFRJ. 2013. Disponível em: $<$ http://gl.globo.com/rio-de-janeiro/noticia/2013/10/ aparelhos-de-ultima-geracao-estao-encaixotados-no-
EMH do SUS, exceto para as instituições da Hemorrede. As ações dos órgãos ligados ao MS desenvolvem ações desarticuladas, por exemplo, a ferramenta CNES não foi citada na maioria das publicações.

Embora haja um investimento crescente para a adequação tecnológica dos SUS, a falta de um sistema de gestão eficiente desse patrimônio afeta o desenvolvimento das políticas de saúde. Notícias na mídia sobre equipamentos não instalados devido à infraestrutura inadequada são frequentes e afetam diretamente o acesso do paciente aos serviços do SUS.

Medidas que reforcem a responsabilização dos gestores poderiam levar à maior preocupação com a necessidade de planejamento das ações e com o desperdício de recursos públicos. $\mathrm{O}$ aperfeiçoamento dos processos de alocação de recursos tecnológicos que considerem a importância da redução das desigualdades geográficas e sociais é condição necessária para a obtenção da equidade implícita na legislação brasileira, no que se refere ao setor saúde. -hospital-da-ufrj.html>. Acesso em: 15 abr. 2013.

BRASIL. Constituição (1988). Constituição da República Federativa do Brasil. Brasília, DF: Senado Federal: Centro Gráfico, 1988. 292 p.

Lei ${ }^{\circ}$ 8.689, de 27 de julho de 1993. Dispõe sobre a extinção do Instituto Nacional de Assistência Médica da Previdência Social (Inamps) e dá outras providências. Diário Oficial [da] União. Brasília, DF, 27 jul. 1993. Disponível em: <http://www.planalto.gov.br/ ccivil_03/leis/L8689.htm>. Acesso em: 20 jan. 2015.

Ministério da Saúde. Portaria MS no 1.101, de 12 de junho de 2002. Dispõe sobre os parâmetros de cobertura assistencial no âmbito do Sistema Único de Saúde - SUS. Diário Oficial [da] União 2002, 13 jun. 2002. 
Ministério da Saúde. Portaria MS n ${ }^{0} 2.481$, de 2 de outubro de 2007 - Institui o Sistema de Apoio à Elaboração de Projetos de Investimentos em Saúde - SOMASUS. Diário Oficial [da] União. Brasília, DF, 2 out. 2007. Disponível em: <http://bvsms.saude.gov.br/ bvs/saudelegis/gm/2007/prt2481_02_10_2007.html>. Acesso em: 15 abr. 2013.

Ministério da Saúde. Portaria MS nº 2.915, de 12 de dezembro de 2011 - Institui a Rede Brasileira de Avaliação de Tecnologias em Saúde (REBRATS). Diário Oficial [da] União. Brasília, DF, 12 dez. 2011a. Disponível em: <http://bvsms.saude.gov.br/bvs/saudelegis/gm/2011/prt2915_12_12_2011.html>. Acesso em: 15 abr. 2013.

Ministério da Saúde. Portaria MS no 263, de 18 de fevereiro de 2011 - Institui Grupo de Assessoramento Técnico em Gestão de Equipamentos dos Serviços de Hemoterapia e Hematologia Públicos, visando à elaboração de propostas e pactuação de ações nas áreas de Gestão de Equipamentos nos Serviços de Hemoterapia e Hematologia Públicos. Diário Oficial [da] União. Brasília, DF, 18 fev. 2011b. Disponível em: <http://bvsms.saude.gov.br/bvs/saudelegis/gm/2011/prt0263_18_02_2011.html>. Acesso em: 15 abr. 2013.

Ministério da Saúde. Portaria. Portaria MS $\mathrm{n}^{\mathrm{o}} 2.510$, de 19 de dezembro de 2005 - Instituiu comissão interinstitucional para elaboração da Política de Gestão de Tecnologias em Saúde, sob coordenação da Secretaria de Ciência, Tecnologia e Insumos Estratégicos (SCTIE). Diário Oficial [da] União. Brasília, DF, 19 dez. 2005. Disponível em: <http:// www.funasa.gov.br/site/wp-content/files_mf/ Pm_2510_2005.pdf >. Acesso em: 15 abr. 2013.

Ministério da Saúde. Secretaria de Assistência à Saúde. Relatório de gestão SAS quadriênio 1998-2001. Brasília, DF: Ministério da Saúde, 2002, 220 p.

. Ministério da Saúde. Secretaria de Atenção à Saúde. Cursos de Especialização em infraestrutura física de serviços de saúde: engenharia clínica, técnico em manutenção de equipamentos, artífices em manutenção de equipamentos, física médica e arquitetura de sistemas de saúde. Brasília, DF: Mimeo, 1992.

Ministério da Saúde. Secretaria de Atenção à Saúde. Departamento de atenção especializada. Guia para elaboração do Plano de gestão de equipamentos para serviços de Hematologia e Hemoterapia. Brasília, DF: Ministério da Saúde, 2012. Disponível em: <http:// bvsms.saude.gov.br/bvs/publicacoes/elaboracao_plano_gestao_equipamentos_servicos_hematologia_hemoterapia>. Acesso em: 10 abr. 2015.

. Ministério da Saúde. Secretaria de Atenção à

Saúde. Programa de equipamentos odonto-médico-hospitalares - PROEQUIPO. Brasília, DF: Mimeo, 1991.

Ministério da Saúde. Secretaria de Ciência, Tecnologia e Insumos Estratégicos. Departamento de Ciência e Tecnologia. Política Nacional de Gestão de Tecnologias em Saúde - PNGTS. Brasília, DF: Ministério da Saúde, 2010a.

Ministério da Saúde. Secretaria de Ciência, Tecnologia e Insumos Estratégicos. Departamento de Ciência e Tecnologia. Diretrizes metodológicas: elaboração de estudos para avaliação de equipamentos médicos assistenciais. Brasília, DF: Ministério da Saúde, 2013a. Disponível em: <http://bvsms.saude.gov.br/bvs/publicacoes/diretrizes_metodologicas_elaboracao_estudos. pdf>. Acesso em: 18 abr. 2015.

Ministério de Saúde. Secretaria Executiva.

Departamento de Economia da Saúde e

Desenvolvimento. Programação Arquitetônica de Unidades Funcionais de Saúde. SOMASUS - Sistema de Apoio à Elaboração de Projetos de Investimentos em Saúde. Brasília, DF: Ministério da Saúde, 2011c. (Atendimento Ambulatorial e Atendimento Imediato, v.1). Disponível em: <http://bvsms.saude.gov.br/bvs/ publicacoes/programacao_arquitetonica_somasus_ vl.pdf> Acesso em: 16 abr. 2015.

Ministério de Saúde. Secretaria Executiva. Departamento de Economia da Saúde e Desenvolvimento. Programação Arquitetônica de Unidades Funcionais de Saúde. SOMASUS - Sistema de Apoio à Elaboração de Projetos de Investimentos em Saúde. Brasília, DF: Ministério da Saúde, 2013b. 
(Programação Arquitetônica de Unidades Funcionais de Saúde, v. 2). Disponível em: <http://bvsms.saude. gov.br/bvs/publicacoes/soma_sus_sistema_apoio_elaboracao_vol2.pdf> Acesso em: 18 abr. 2015.

Ministério de Saúde. Secretaria Executiva.

Departamento de Economia da Saúde e

Desenvolvimento. Programação Arquitetônica de Unidades Funcionais de Saúde. SOMASUS - Sistema de Apoio à Elaboração de Projetos de Investimentos em Saúde. Brasília, DF: Ministério da Saúde, 2013c. (Programação Arquitetônica de Unidades Funcionais de Saúde, v. 3). Disponível em: < http://bvsms.saude. gov.br/bvs/publicacoes/soma_sus_sistema_apoio_elaboracao_vol3.pdf $>$. Acesso em: 10 abr. 2015.

Resolução da Diretoria Colegiada no 2 , de 25

de janeiro de 2010 - Dispõe sobre o gerenciamento de tecnologias em saúde em estabelecimentos de saúde. Diário Oficial [da]União. Brasília, DF, 25 jan. 2010b. Disponível em: <http://bvsms.saude.gov.br/bvs/saudelegis/anvisa/2010/res0002_25_01_2010.html>. Acesso em: 16 abr. 2013

CALIL, S. J. Análise do Setor de Saúde no Brasil na área de equipamentos médico-hospitalares. In.: NEGRI, B.; Di Giovanni, G. (Org.). Brasil: radiografia da saúde. Campinas, SP: Unicamp, 2001, p. 91-122.

GALVAN, P. Clinical Engineering in Paraguay. In: DYRO, J. (Org). The Clinical Engineering Handbook. New York: Elsevier, 2004, p. 84-86.

GENTLES, W. M. Clinical Engineering in Canada. In: DYRO, J. (Org). The Clinical Engineering Handbook. New York: Elsevier, 2004, p. 62-64.

GOMES, J. Clinical Engineering in Equador. In: DYRO, J. (Org). The Clinical Engineering Handbook. New York: Elsevier, 2004, p. 93-95.

GUIMARÃES, J. M. C.; GONDIM, G. M. de M. O papel da politécnica na formação profissional de técnicos de nível médio envolvidos na área de manutenção predial e de equipamentos em estabelecimentos assistenciais de saúde (EAS) [internet], 2008. Disponível em: <http:// www.senept.cefetmg.br/galerias/Anais_2010/Artigos/ GT3/O_PAPEL_DA_POLITECNIA.pdf. >. Acesso em: 20 jan. 2015.

GUTIÉRREZ, J. E. V. Clinical Engineering in Colombia. In: DYRO, J. (Org.). The Clinical Engineering Handbook. New York: Elsevier, 2004, p. 72-77.

GUTIERREZ, M. S. A oferta de tomógrafo computadorizado para o tratamento do acidente vascular cerebral agudo, no Brasil, sob o ponto de vista das desigualdades geográficas e sociais. 2009. 61f. Dissertação (Mestrado profissional em Saúde Pública) - Escola Nacional de Saúde Pública Sergio Arouca, Brasília, DF, 2009.

\section{INSTITUTO BRASILEIRO DE GEOGRAFIA}

E ESTATÍSTICA (IBGE). Estatísticas da Saúde Assistência Médico-Sanitária. Rio de Janeiro: IBGE, 2010. $167 \mathrm{p}$.

TOSCAS, F. S. Estudo dos equipamentos médico-hospitalares e materiais permanentes financiáveis pelo SUS nos anos de 2010, 2011 e 2012 através do sistema de pagamento fundo a fundo e GESCON. 2013. Trabalho de conclusão de curso (Especialização em Engenharia Biomédica) - Faculdade Gama, Distrito Federal, DF, 2013.

SUMALGY, E. N.; SUMALGY, M. Clinical Engineering in Mozambique. In: DYRO, J. (Org.). The Clinical Engineering Handbook. New York: Elsevier, 2004, p. 93-96.

\footnotetext{
Recebido para publicação em junho de 2014

Versão final em abril de 2015

Conflito de interesse: inexistente

Suporte financeiro: não houve
} 